Pacific Journal of Mathematic 


\title{
STRUCTURE HYPERGROUPS FOR MEASURE ALGEBRAS
}

\section{Charles F. DunkL}

\begin{abstract}
An abstract measure algebra $A$ is a Banach algebra of measures on a locally compact Hausdorff space $X$ such that the set of probability measures in $A$ is mapped into itself under multiplication, and if $\mu$ is a finite regular Borel measure on $X$ and $\mu<<\nu \in A$ then $\mu \in A$. If $A$ is commutative then the spectrum of $A, \Delta_{A}$, is a subset of the dual of $A, A^{*}$, which is a commutative $W^{*}$-algebra. In this paper conditions are given which insure that the weak-* closed convex hull of $\Delta_{A}$, or of some subset of $\Delta_{A}$, is a subsemigroup of the unit ball of $A^{*}$. This statement implies the existence of certain bypergroup structures. An example is given for which the conditions fail.

The theory is then applied to the measure algebra of a compact $P^{*}$-hypergroup, for example, the algebra of central measures on a compact group, or the algebra of measures on certain homogeneous spaces. A further hypothesis, which is satisfied by the algebra of measures given by ultraspherical series, is given and it is used to give a complete description of the spectrum and the idempotents in this case.
\end{abstract}

A hypergroup is a locally compact space on which the space of finite regular Borel measures has a commutative convolution structure preserving the probability measures. The spectrum of the measure algebra of a locally compact abelian group is the semigroup of all continuous semicharacters of a commutative compact topological semigroup (Taylor [7], or see [2, Ch. 1]). In this paper we consider the spectrum of an abstract measure algebra and investigate the question of whether the spectrum or some subset of it has a hypergroup structure.

Section 1 of the paper contains a general theorem on the existence of hypergroup structures on the spectrum of an abstract measure algebra. The fact that the dual space of an appropriate space of measures is a commutative $W^{*}$-algebra is of basic importance in the proof of this theorem. This section also contains an example of a compact hypergroup whose measure algebra does not satisfy the hypotheses of the theorem.

In $\S 2$ we recall the definition of a compact $P^{*}$-hypergroup from a previous paper [1] and apply the main theorem of $\S 1$ to this situation. The result is that the closure of the set of characters of the hypergroup in the spectrum is a compact semitopological hypergroup and is a set of characters on another compact semitopological hypergroup. 
Section 3 defines a class of $P^{*}$-hypergroups of which ultraspherical series form a particular example. A complete description of the spectrum and the idempotents of the measure algebra is given. The results are much like those which Ragozin [6] obtained for the algebra of central measures on a compact simple Lie group.

1. The general situation. We will use the following notation; for a locally compact Hausdorff space $X, C^{B}(X)$ is the space of bounded continuous functions on $X, C_{0}(X)$ is the space $\left\{f \in C^{B}(X): f\right.$ tends to 0 at $\infty\}, M(X)$ is the space of finite regular Borel measures on $X, M_{p}(X)$ is the set $\{\mu \in M(X): \mu \geqq 0, \mu X=1\}$ (the probability measures), $\delta_{x}$ is the unit point mass at $x \in X$, and $M(X)^{*}$ is the dual space of $M(X)$. If $X$ is compact we write $C(X)$ for $C^{B}(X)$. We let $w^{*}$ denote either of the topologies $\sigma\left(M(X), C_{0}(X)\right)$ or $\sigma\left(M(X)^{*}, M(X)\right)$.

Note that $M(X)^{*}$ may be interpreted as the space of generalized functions on $X$, (the projective limit of the spaces $\left\{L^{\infty}(X, \mu): \mu \in M_{p}(X)\right\}$ ordered by absolute continuity) and is thus seen as a commutative $W^{*}$-algebra (see [2, p. 9]). We will write $f \rightarrow \bar{f}\left(f \in M(X)^{*}\right)$ for the involution, $f \cdot \mu$ for the action of $M(X)^{*}$ on $M(X)$, and $\langle\mu, f\rangle$ for the pairing of $M(X)$ and $M(X)^{*},\left(\mu \in M(X), f \in M(X)^{*}\right)$. Note $\langle f \cdot \mu, g\rangle=$ $\langle\mu, f g\rangle$ for $f, g \in M(X)^{*}, \mu \in M(X)$, and $\langle\mu, 1\rangle=\int_{X} d \mu$. The unit ball $B$ (the set $\{f:\|f\| \leqq 1\}$ ) of $M(X)^{*}$ is $w^{*}$-compact and is a commutative semitopological semigroup under multiplication and the $w^{*}$-topology. We will be concerned with compact convex subsemigroups of $B$.

Suppose there is given for each $x, y \in X$ a measure $\lambda(x, y) \in M_{p}(X)$ such that for each $f \in C_{0}(X)$ the map $(x, y) \mapsto \int_{X} f d \lambda(x, y)$ is separately continuous. Then for each $\mu, \nu \in M(X)$ the function

$$
x \mapsto \int_{X} \int_{X} f d \lambda(x, y) d \nu(y)
$$

is continuous and

$$
\int_{X} d \mu(x) \int_{X} d \nu(y) \int_{X} f d \lambda(x, y)=\int_{X} d \nu(y) \int_{X} d \mu(x) \int_{X} f d \lambda(x, y) \text {. }
$$

This fact was proved by Glicksberg [3]. We will use this to define semitopological hypergroups.

Definition 1.1. A locally compact space $H$ is called a semitopological hypergroup if there is a map $\lambda: H \times H \rightarrow M_{p}(H)$ with the following properties:

(1) $\lambda(x, y)=\lambda(y, x),(x, y \in H)$, (commutativity);

(2) for each $f \in C_{0}(H)$ the map $(x, y) \mapsto \int_{H} f d \lambda(x, y)$ is separately continuous, $(x, y \in H)$; 
(3) the convolution on $M(H)$ defined implicitly by

$$
\int_{H} f d(\mu * \nu)=\int_{H} d \mu(x) \int_{H} d \nu(y) \int_{H} f d \lambda(x, y),\left(\mu, \nu \in M(H), f \in C_{0}(H)\right)
$$

is associative, (note $\delta_{x} * \delta_{y}=\lambda(x, y),(x, y \in H)$ ).

If there is a point $e \in H$ such that $\lambda(e, x)=\delta_{x},(x \in H)$, then $e$ is called the identity of $H$. A bounded continuous function $\phi$ on $H$ such that $\int_{H} \phi d \lambda(x, y)=\phi(x) \phi(y),(x, y \in H)$, is called a character of $H$.

If $H$ is a compact semitopological hypergroup then it is easily shown that convolution on $M(H)$ is separately $w^{*}$-continuous, and that $M_{p}(H)$ is a compact commutative semitopological affine semigroup ("affine" means $\mu *\left(s_{1} \nu_{1}+s_{2} \nu_{2}\right)=s_{1}\left(\mu * \nu_{1}\right)+s_{2}\left(\mu * \nu_{2}\right)$ for $s_{1}, s_{2} \geqq 0, s_{1}+$ $s_{2}=1, \mu, \nu_{1}, \nu_{2} \in M_{p}(H)$ ). The converse to the latter holds (Pym [4] proved a form of this statement; we will give a proof of it in the present context).

Proposition 1.2. Let $H$ be a compact space and suppose $M_{p}(H)$ is a commutative semitopological affine semigroup (in the $w^{*}$-topology), then $H$ can be given the structure of a compact semitopological hypergroup, so that convolution restricted to $M_{p}(H)$ gives the original semigroup structure.

Proof. Let $*$ denote the semigroup operation on $M_{p}(H)$. This operation extends uniquely to $M(H)$, and $M(H)$ becomes a commutative Banach algebra. For each $x, y \in H$ let $\lambda(x, y)=\delta_{x} * \delta_{y} \in M_{p}(H)$. Now we must show that $\lambda$ satisfies Definition 1.1, and the convolution induced by $\lambda$ is the same as the given. By hypothesis, the function $T f(x, y)=\int_{H} f d \lambda(x, y)=\int_{H} f d\left(\delta_{x} * \delta_{y}\right)$ is separately continuous $(x, y \in H)$. Glicksberg's result [3] shows that $x \mapsto \int_{H} T f(x, y) d \mu(y)$ is continuous

for each $\mu \in M(H)$. Let $\mu, \nu$ be finitely supported (discrete) measures in $M_{p}(H)$, then by an easy computation we have

$$
\int_{H} \int_{H} T f(x, y) d \mu(x) d \nu(y)=\int_{H} f d \mu * \nu, \quad(f \in C(H)) .
$$

For fixed $\nu$ the set of $\mu$ for which this identity holds is $w^{*}$-closed. Thus the identity holds for all $\mu \in M_{p}(H)$, all finitely supported $\nu \in$ $M_{p}(H)$. Repeat the argument to show the identity holds for all $\nu \in M_{p}(H)$.

It is convenient to isolate the following situation as a lemma. 
Lemma 1.3. Suppose $X$ is a locally compact space, $S$ is a completely regular Hausdorff space, and there is a bounded linear map $j: M(X) \rightarrow C^{B}(S)$ with the following properties (we will write $\|\mu\|_{S}$ for $\sup \{|j \mu(s)|: s \in S\}$ :

(1) $\|j\|=1$;

(2) there exists $\iota \in M_{p}(X)$ such that $j \iota=1$ (the constant function);

(3) $\left\|j_{1} s \cdot \mu\right\|_{S} \leqq\|\mu\|_{s}$, where $j_{1} s \in M(X)^{*}$ is defined by $\left\langle\mu, j_{1} s\right\rangle=$ $j \mu(s),(s \in S, \mu \in M(X))$.

Then the $w^{*}$-closed convex hull of $j_{1} S$, denoted by $w^{*}$ co $\left(j_{1} S\right)$, is a compact (semitopological) subsemigroup of $B$, the unit ball in $M(X)^{*}$. Each map $f \mapsto\left\langle\delta_{x}, f\right\rangle,(x \in H)$, is an affine semicharacter on $w^{*}$ co $\left(j_{1} S\right)$. Further, if $S$ is compact and $j M(X)$ is sup-norm dense in $C(S)$, then $S$ has a semitopological hypergroup structure, and the functions $\left\{j \delta_{x}: x \in X\right\}$ are characters of $S$.

Proof. Let $S_{1}$ be a compactification of $S$ such that $j M(X) \subset C\left(S_{1}\right)$, and let $j^{*}$ denote the adjoint map: $M\left(S_{1}\right) \rightarrow M(X)^{*}$,

$$
\text { (given by } \left.\left\langle\mu, j^{*} \lambda\right\rangle=\int_{S_{1}} j \mu d \lambda, \mu \in M(X), \lambda \in M\left(S_{1}\right)\right) \text {. }
$$

Denote $w^{*} \operatorname{co}\left(j_{1} S\right)$ by $S_{c}$. We claim $j^{*} M_{p}\left(S_{1}\right)=S_{c}$. The map $j^{*}$ is $w^{*}$-continuous $M\left(S_{1}\right) \rightarrow M(X)^{*}$ thus $j^{*}$ maps $w^{*}$ co $\left\{\delta_{s}: s \in S_{s}\right\}$ (in $M\left(S_{1}\right)$ ) into $S_{c}$. That is, $j^{*} M_{p}\left(S_{1}\right) \subset S_{c}$. Conversely let $f \in S_{c}$, then there exists a net $\left\{f_{\alpha}\right\} \subset \operatorname{co}\left(j_{1} S\right)$, (the convex hull of $j_{1} S$ ) so that $f_{\alpha} \underset{\alpha}{\longrightarrow} f\left(w^{*}\right)$. But for each $\alpha$ there exists a finitely supported $\lambda_{\alpha} \in M_{p}\left(S_{1}\right)$ so that $j^{*} \lambda_{\alpha}=f_{\alpha}$. By the $w^{*}$-compactness of $M_{p}\left(S_{1}\right)$ there exists $\lambda \in M_{p}\left(S_{1}\right)$ so that $j^{*} \lambda=f$. Thus $j^{*} M_{p}\left(S_{1}\right)=S_{c}$.

We observe for $g \in M(X)^{*}$ that $g \in S_{c}$ if and only if $|\langle\mu, g\rangle| \leqq$ $\|\mu\|_{s},(\mu \in M(X))$ and $\langle\iota, g\rangle=1$. The latter condition and the HahnBanach and Riesz theorems imply that there exists $\lambda \in M_{p}\left(S_{1}\right)$ so that $j^{*} \lambda=g$. We now show for $s \in S, \lambda \in M_{p}\left(S_{1}\right)$ that $\left(j_{1} s\right)\left(j^{*} \lambda\right) \in S_{c}$. Indeed for $\mu \in M(X)$, |

$$
\begin{aligned}
\left\langle\mu,\left(j_{1} s\right)\left(j^{*} \lambda\right)\right\rangle \mid & =\left|\left\langle j_{1} s \cdot \mu, j^{*} \lambda\right\rangle\right| \\
& =\left|\int_{S_{1}} j\left(j_{1} s \cdot \mu\right) d \lambda\right| \leqq\left\|j_{1} s \cdot \mu\right\|_{s} \leqq\|\mu\|_{s} .
\end{aligned}
$$

Also $\left\langle\iota,\left(j_{1} s\right)\left(j^{*} \lambda\right)\right\rangle=\left\langle j_{1} s \cdot \iota, j^{*} \lambda\right\rangle=\left\langle\iota, j^{*} \lambda\right\rangle=1$, (note $j_{1} s \cdot \iota=\iota$, since $\left\|j_{1} s\right\| \leqq 1,\left\langle\iota, j_{1} s\right\rangle=j \iota(s)=1$ and $\left.\iota \in M_{p}(X)\right)$. Thus $\left(j_{1} s\right)\left(j^{*} \lambda\right) \in S_{c}$ and we conclude from the separate $w^{*}$-continuity of multiplication that $S_{c} S_{c} \subset S_{c}$; so $S_{c}$ is a subsemigroup of $B$.

For each $x \in X, f \in M(X)^{*}$ we have that $f \cdot \delta_{x}=\left\langle\delta_{x}, f\right\rangle \delta_{x}$ so the maps $f \mapsto\left\langle\delta_{x}, f\right\rangle$ are affine semicharacters of $S_{c}$. 
Now suppose that $S$ is a compact and $j M(X)$ is norm dense in $C(S)$. Then $j^{*}$ maps $M_{p}(S)$ one-to-one, $w^{*}$-continuous, and onto $S_{c}$. Thus $M_{p}(S)$ with the $w^{*}$-topology is homeomorphic to $S_{c}$. We define a semigroup structure on $M_{p}(S)$ by using this isomorphism (that is, for $\lambda, \nu \in M_{p}(S)$ define $\left.\lambda * \nu=\left(j^{*}\right)^{-1}\left(\left(j^{*} \lambda\right)\left(j^{*} \nu\right)\right)\right)$. Thus $M_{p}(S)$ is a commutative affine $w^{*}$-semitopological semigroup. By Proposition $1.2 S$ is a compact semitopological hypergroup. Further for $x \in X, \lambda \in M(S)$, $\int_{S}\left(j \delta_{x}\right) d \lambda=\left\langle\delta_{x}, j^{*} \lambda\right\rangle$, which shows that $j \delta_{x}$ is a character of $S$.

Note that in the lemma $M(X)$ may be replaced by an $L$-subspace $A$ of $M(X)$, (that is, $A$ is a closed subspace of $M(X)$ and $\mu \in M(X)$ and $\mu<<\nu \in A$ implies $\mu \in A)$. The dual of $A$ is a $w^{*}$-closed ideal in $M(X)^{*}$ and so is itself a commutative $W^{*}$-algebra. However, the point masses $\delta_{x}$ may not be in $A$.

Definition 1.4. Suppose $X$ is a locally compact Hausdorff space and $A$ is an $L$-subspace of $M(X)$. Say $A$ is an abstract measure algebra if it is a Banach algebra in the measure norm, and $A_{p} A_{p} \subset A_{p}$ (where $A_{p}=A \cap M_{p}(X)$ ). We say $A$ has an identity if there exists an algebra identity $\iota \in A_{p}$. If $A$ is commutative we let $\Delta_{A}$ denote the spectrum (maximal ideal space) of $A$, considered as a subset of the unit ball of the dual $A^{*}$ of $A$. Further $\tilde{\mu}$ denotes the Gelfand transform of $\mu \in A$, so $\tilde{\mu} \in C_{0}\left(\Delta_{A}\right)$.

THEOREM 1.5. Suppose $A$ is a commutative abstract measure algebra with identity $\iota$, and $E$ is a $w^{*}$-closed subset of $\Delta_{A}$ with the following properties: (1) $1 \in E$; (2) $f \in E$ implies $\bar{f} \in E$; (3) $g \in E, \mu \in A$ imply $\left\|(g \cdot \mu)^{\sim}\right\|_{E} \leqq\|\tilde{\mu}\|_{E}$, (where $\left.\|\tilde{\mu}\|_{E}=\sup \{|\tilde{\mu}(f)|: f \in E\}\right)$. Then the norm-closed linear span of $w^{*}$ co $E$ is isomorphic to $C(Y)$, where $Y$ is a compact semitopological hypergroup with an identity, and the natural map $\sigma: A \rightarrow M(Y)$ is a homomorphism with $w^{*}$-dense range. Further $\sigma \iota=\delta_{e}$, where $e$ is the identity in $Y$. If $A$ contains a point mass $\delta_{x}$, then $\sigma \delta_{x}$ is a point mass in $Y$. The set $E$ considered as a subset of $C(Y)$ consists of characters of $Y$.

Proof. The Gelfand transform maps $A \rightarrow C(E)$. By Lemma 1.3 $w^{*} \operatorname{co}(E)$ is closed under multiplication. Thus the norm closure of $\operatorname{sp}\left(w^{*} \operatorname{co}(E)\right)$ is a self-adjoint closed subalgebra of $A^{*}$, hence is isomorphic to $C(Y)$, ( $Y$ is its spectrum). We define the natural map $j: M(E) \rightarrow C(Y)$ so that $\langle\mu, j \lambda\rangle=\int_{E} \tilde{\mu} d \lambda,(\mu \in A, \lambda \in M(E))$; note $j \lambda \epsilon$ $C(Y) \subset A^{*}$. Observe $j \delta_{1}=1$, and $j M_{p}(E)=w^{*}$ co $(E)$. We show that $j$ satisfies the hypotheses of Lemma 1.3. Note that $\|j \lambda\|_{Y}$ is given by 


$$
\begin{aligned}
\|j \lambda\|_{Y} & =\sup \{|\langle\mu, j \lambda\rangle|: \mu \in A,\|\mu\| \leqq 1\} \\
& =\sup \left\{\left|\int_{E} \tilde{\mu} d \lambda\right|: \mu \in A,\|\mu\| \leqq 1\right\} .
\end{aligned}
$$

Let $y \in Y$ and define $j_{1}: Y \rightarrow M(E)^{*}$ by $\left\langle\lambda, j_{1} y\right\rangle=j \lambda(y),(\lambda \in M(E))$. For $\mu \in A, \lambda \in M(E)$ we have

$$
\left\langle\mu, j\left(j_{1} y \cdot \lambda\right)\right\rangle=\int_{E} \tilde{\mu} d\left(j_{1} y \cdot \lambda\right)=\left\langle\tilde{\mu} \cdot \lambda, j_{1} y\right\rangle=j(\tilde{\mu} \cdot \lambda)(y) .
$$

Thus

$$
\left\|j_{1} y \cdot \lambda\right\|_{Y} \leqq \sup \left\{\|j(\tilde{\mu} \cdot \lambda)\|_{Y}: \mu \in A,\|\mu\| \leqq 1\right\}
$$

Now

$$
\begin{aligned}
\|j(\tilde{\mu} \cdot \lambda)\|_{Y} & =\sup \{|\langle\nu, j(\tilde{\mu} \cdot \lambda)\rangle|: \nu \in A,\|\nu\| \leqq 1\} \\
& =\sup \left\{\left|\int_{E} \tilde{\nu} \tilde{\mu} d \lambda\right|: \nu \in A,\|\nu\| \leqq 1\right\} \\
& \leqq \sup \left\{\|\nu\|\|\mu\|\|j \lambda\|_{Y}: \nu \in A,\|\nu\| \leqq 1\right\} \\
& =\|\mu\|\|j \lambda\|_{Y},
\end{aligned}
$$

(since $\tilde{\nu} \tilde{\mu}=(\nu \mu)^{\sim}$ and $\left.\|\nu \mu\| \leqq\|\nu\|\|\mu\|\right)$. Thus $\left\|j_{1} y \cdot \lambda\right\|_{Y} \leqq\|\lambda\|_{Y}$. Further $j M(E)=\operatorname{sp}\left(w^{*}\right.$ co $\left.E\right)$ is dense in $C(Y)$, so by Lemma $1.3 Y$ is a compact semitopological hypergroup. Note that $E \subset C(Y)$ consists of characters of $Y$.

Let $\sigma$ be the natural map $A \rightarrow M(Y)$. Clearly $\sigma A$ is $w^{*}$-dense in $M(Y)$. Further the convolution on $M(Y)$ is defined in terms of multiplication in $M(E)^{*}$, but the map $A \rightarrow C(E) \subset M(E)^{*}$ is a homomorphism, so $\sigma$ is a homomorphism.

Since $i=1$ on $E$ we have $\langle\iota, f\rangle=1$ for all $f \in w^{*}$ co $E$. For $f, g \in w^{*} \operatorname{co}(E),\langle\iota, f g\rangle=1=\langle\iota, f\rangle\langle\iota, g\rangle$ (since $f g \in w^{*} \operatorname{co} E$ ) thus $f \rightarrow\langle\iota, f\rangle$ is multiplicative and norm bounded on $\operatorname{sp}\left(w^{*} \operatorname{co}(E)\right)$, so there exists a unique point $e \in Y$ so that $\langle\iota, f\rangle=f(e),(f \in C(Y))$. Thus $\sigma \iota=\delta_{e}$ and $e$ is the identity of $Y$. If there is a point mass $\delta_{x} \in A$ then $f \rightarrow\left\langle\delta_{x}, f\right\rangle$ is multiplicative on $A^{*}$, so $\sigma \delta_{x}$ is a point mass in $Y$.

It would be interesting to know whether $Y$ has any characters other than the elements of $E$, but the answer is presently unknown to the author. If $\Delta_{A}$ has the properties specified for $E$, then the set characters of $Y$ is $A_{A}$, since $\sigma A$ is $w^{*}$ dense in $M(Y)$ and characters of $Y$ give multiplicative linear functionals on $M(Y)$.

This line of investigation was motivated partly by Taylor's work [7] on structure semigroups of convolution measure algebras. Pym [5] has a result similar to Theorem 1.5 for the spectrum of a com- 
mutative Banach measure algebra $M(X)$ in which multiplication is separately $w^{*}$-continuous and the map $\mu \mapsto f \cdot \mu$ is bounded in the spectral norm $\left(\mu \mapsto\|\tilde{\mu}\|_{\infty}\right)$, for each $f \in \Delta_{M(X)}$.

A compact hypergroup $H$ is defined by Definition 1.1 with "separately continuous" in condition (2) replaced by "jointly continuous". We write $\hat{H}$ for the set of characters of $H$, and $\Delta_{H}$ for the spectrum of $M(H)$. For $\mu \in M(H), \phi \in \hat{H}$, let $\hat{\mu}(\phi)=\int_{H} \bar{\phi} d \mu$. In the sequel we will refer to [1] for necessary details.

We will now construct a compact hypergroup $H$ for which neither $\Delta_{H}$ nor the closure of $\hat{H}$ in $\Delta_{H}$ satisfy the hypotheses of Theorem 1.5.

EXAMPLE 1.6. There exists a compact hypergroup $H$ and $\psi \in \kappa \hat{H}$ (the closure of $\hat{H}$ in $\Delta_{H}$ ) such that $\mu \mapsto \psi \cdot \mu,(\mu \in M(H))$, is bounded in neither the $\|\tilde{*}\|_{\infty}$ nor the $\|\cdot\|_{\infty}$ norm.

Proof. Let $H_{1}$ be the finite hypergroup described in Example 4.6 of [1]. Briefly the points of $H_{1}$ correspond to rows of the matrix

$$
\begin{gathered}
r_{1} \\
r_{2} \\
r_{2}
\end{gathered}\left[\begin{array}{ccc}
1 & \phi_{1} & \phi_{2} \\
1 & -1 / 2 & 0 \\
1 & 1 / 4 & 0
\end{array}\right]
$$

and multiplication is pointwise. That is, the columns correspond to the characters of $H_{1}$. Note that $\phi_{1}^{2}=(1 / 8)\left(\phi_{0}-2 \phi_{1}+9 \phi_{2}\right)$. Let $\nu$ be the measure $\delta_{e}+\delta_{r_{1}}-2 \delta_{r_{2}}$ on $H_{1}$, then $\widetilde{\nu}\left(\phi_{0}\right)=0, \widetilde{\nu}\left(\phi_{1}\right)=0$, and $\widetilde{\nu}\left(\phi_{2}\right)=1$.

Let $H$ be the Tikhonov product $\prod_{n=1}^{\infty} H_{1}$, so $H$ is a compact hypergroup. For $n=1,2, \cdots$, let $H_{n}=\prod_{i=1}^{n} H_{1}$. We identify $M\left(H_{n}\right)$ with a subalgebra of $M(H)$ under the map

$$
\int_{H} f d \sigma \mu=\int_{H_{n}} f\left(x_{1}, \cdots, x_{n}, e, e, \cdots\right) d \mu\left(x_{1}, \cdots, x_{n}\right),
$$

$\left(f \in C(H), \mu \in M\left(H_{n}\right)\right)$. By a multi-index $I$ we mean a sequence $I=$ $\left(i_{1}, i_{2}, \cdots\right)$ where $i_{s}=0,1,2$ and $i_{s}=0$ for all but finitely many $s$. For a multi-index $I$ let $\phi_{I}(x)=\phi_{i_{1}}\left(x_{1}\right) \phi_{i_{2}}\left(x_{2}\right) \cdots$, then $\phi_{I} \in \hat{H}$. Let $\nu_{n}=$ $\nu \times \cdots \times \nu(n$ times $)$, an element of $M\left(H_{n}\right)$, and let $\mu_{n}=\sigma \nu_{n} \in M(H)$. The spectrum of $M\left(H_{n}\right)$ is isomorphic to $S_{n}=\left\{\phi_{I}: I\right.$ multi-index, $i_{s}=0$ for $s>n\}$. Thus the spectral norm of a measure in $M\left(H_{n}\right)$ (or $\sigma M\left(H_{n}\right)$ ) is realized on $S_{n}$. Let $\psi_{n}^{m} \in \hat{H}$ be given by $\psi_{n}^{(m)}(x)=\phi_{m}\left(x_{1}\right) \cdots \phi_{m}\left(x_{n}\right)$ $(x \in H, m=1,2)$. We claim $\left\|\tilde{\mu}_{n}\right\|_{\infty}=\left\|\hat{\mu}_{n}\right\|_{\infty}=1$, in fact for $\phi_{I} \in S_{n}$, $\left\langle\mu_{n}, \phi_{I}\right\rangle=\prod_{s=1}^{n}\left\langle\nu, \phi_{i_{s}}\right\rangle=0$ if $\phi_{I} \neq \psi_{n}^{(2)}$, and $\left\langle\mu_{n}, \psi_{n}^{(2)}\right\rangle=1$. Let $m \geqq n$, then $\left\langle\psi_{m}^{(1)} \cdot \mu_{n}, \psi_{n}^{(1)}\right\rangle=(9 / 8)^{n}$. Indeed $\left\langle\psi_{m}^{(1)} \cdot \mu_{n}, \psi_{n}^{(1)}\right\rangle=\int_{H} \psi_{m}^{(1)} \psi_{n}^{(1)} d \mu_{n}=$ $\Pi_{s=1}^{n}\left\langle\nu, \phi_{1} \phi_{1}\right\rangle=(9 / 8)^{n}$. Let $\psi$ be a $w^{*}$-cluster point of $\left\{\psi_{n}^{(1)}\right\}$ in $\Delta_{H}$. 
Then $\left\langle\psi \cdot \mu_{n}, \psi_{n}^{(1)}\right\rangle=(9 / 8)^{n}$ and $\left\|\tilde{\mu}_{n}\right\|_{\infty}=\left\|\hat{\mu}_{n}\right\|_{\infty}=1$, but $\left\|\left(\psi \cdot \mu_{n}\right)^{\sim}\right\|_{\infty} \geqq$ $\left\|\left(\psi \cdot \mu_{n}\right)^{\wedge}\right\|_{\infty} \geqq(9 / 8)^{n}$.

2. $P^{*}$-hypergroups. See [1] for a reference for this section.

Definition 2.1. A compact hypergroup $H$ is called a $P^{*}$-hypergroup if:

(1) there exists an invariant measure $m_{I I} \in M_{p}(H)$ and a continuous involution $x \mapsto x^{\prime},(x \in H)$ such that

$$
\int_{I I}(R(x) f) \bar{g} d m_{H}=\int_{H} f\left(R\left(x^{\prime}\right) g\right)^{-} d m_{H},
$$

and such that $e \in \operatorname{support} \lambda\left(x, x^{\prime}\right),(f, g \in C(H), x \in H),(R(x): C(H) \rightarrow$ $C(H)$ is defined by $\left.R(x) f(y)=\int_{H} f d \lambda(x, y), f \in C(H), x \in H\right)$;

(2) $\hat{H} \hat{H} \subset \operatorname{co} \hat{H}$, that is, for each $\phi, \psi \in \hat{H}$ there exists a nonnegative function $n(\phi, \dot{\psi} ; \cdot)$ on $\hat{H}$ with only finitely many nonzero values such that $\phi(x) \psi(x)=\sum_{\omega \in \hat{H}} n(\phi, \psi ; \omega) \omega(x),(x \in H)$.

Recall from [1] that each subhypergroup $K$ of $H$ is, by definition, closed and is normal $\left(x \in K\right.$ implies $\left.x^{\prime} \in K\right)$, if $H$ is $P^{*}$. Furthermore, $K$ is itself a $P^{*}$-hypergroup with invariant measure $m_{K}$.

Definition 2.2. Let $H$ be a compact $P^{*}$-hypergroup and let $\mu \in M(H)$. Define $\mu^{*} \in M(H)$ by

$$
\int_{H} f d \mu^{*}=\left(\int_{H}\left(f\left(x^{\prime}\right)\right)^{-} d \mu(x)\right)^{-},(f \in C(H)) .
$$

Then $\mu \rightarrow \mu^{*}$ is an algebra involution and $\left(\mu^{*}\right)^{\wedge}(\phi)=(\widehat{\mu}(\phi))^{-},(\phi \in \hat{H})$ (see Theorem $3.5[1]$ ).

Definition 2.3. The set $B(\hat{H})=\{\hat{\mu}: \mu \in M(H)\} \subset C^{B}(\hat{H})$ is a selfadjoint separating algebra of continuous functions on $\hat{H}$ and contains the constants. Let $\kappa \hat{H}$ be the compactification of $\hat{H}$ induced by this algebra. Equivalently $\kappa \hat{H}$ is the spectrum of the sup-norm closure of $B(\hat{H})$, and $\hat{H}$ is a dense open subset.

Theorem 2.4. $\kappa \hat{H}$ is a compact semitopological hypergroup, and $\hat{H}$ is a discrete subhypergroup. Further $\kappa \hat{H}$, as a subset of $\Delta_{H}$ (the spectrum of $M(H)$ ), is $w^{*}$-closed, contains 1 , and is self-adjoint.

Proof. Let $j$ be the bounded linear map: $M(H) \rightarrow C(\kappa \hat{H})$ which is determined by $(j \mu)(\phi)=\hat{\mu}(\phi)=\int_{H} \bar{\phi} d \mu,(\mu \in M(H), \phi \in \hat{H})$. Observe $\|j \mu\|_{\infty}=\|\hat{\mu}\|_{\infty}$. Also $j \delta_{e}=1$. For $\phi, \psi \in \hat{H}, \mu \in M(H)$ we have 


$$
j(\bar{\phi} \cdot \mu)(\psi)=\int_{H} \bar{\phi} \psi d \mu=\sum_{\omega \in \hat{H}} n(\phi, \psi ; \omega) \int_{I I} \bar{\omega} d \mu=\sum_{\omega \in \hat{H}} n(\dot{\phi}, \psi ; \omega) \hat{\mu}(\omega) .
$$

But $|j(\bar{\phi} \cdot \mu)(\psi)| \leqq \sum_{\omega \in \hat{H}} n(\phi, \psi ; \omega)|\hat{\mu}(\omega)| \leqq\|\hat{\mu}\|_{\infty}=\|j \mu\|_{\infty}$. Thus we can apply Lemma 1.3 and obtain that $\kappa \hat{H}$ is a semitopological hypergroup. Further $M_{p}(\kappa \hat{H})$ is isomorphic to $w^{*} \operatorname{co}(\hat{H}) \subset M(H)^{*}$, and the functions $\left\{j \hat{o}_{x}: x \in H\right\}$ are characters of $\kappa \hat{H}$.

We now apply Theorem 1.5 to $\kappa \hat{H}$ and obtain the following:

Theorem 2.5. Suppose $H$ is a compact $P^{*}$-hypergroup, then there exists a compact semitopological hypergroup $Y$ such that $\kappa \hat{H}$ is a set of characters of $Y$, the norm-closed span of $w^{*}$ co $(\hat{H})$ is isomorphic to $C(Y)$, and there is a monomorphism $\sigma: M(H) \rightarrow M(Y)$ with $w^{*}$-dense range.

3. Simple $P^{*}$-hypergroups. In this section $H$ will always denote a compact $P^{*}$-hypergroup. We will describe an additional hypothesis which allows a complete description of $\Delta_{H}$. This hypothesis is realized in the algebra of ultraspherical series (see Example 4.3 [1]). The author suspects that the algebra of central measures on a compact simple Lie group also satisfies the hypothesis.

Recall from [1] that the center of $H, Z(H)$, is $\{x \in H: y \in H$ implies that $\lambda(x, y)$ is a point mass $\}$. Further $Z(H)$ is a compact subgroup of $H$ and is the set $\{x \in H:|\phi(x)|=1,(\phi \in \hat{H})\}$.

Definition 3.1. Let $n$ be a positive integer. Say $H$ has property $S_{n}$ if for each compact set $K \subset H \backslash Z(H)$ the sum $\sum_{\dot{\phi} \in \hat{H}} c(\phi)\left(\sup _{K}|\phi|\right)^{2 n}<$ $\infty$, (where $\left.c(\dot{\phi})=\left(\int_{H}|\phi|^{2} d m_{I I}\right)^{-1}\right)$. (The letter "S" suggests "simple" in the sense that if $K$ is a subhypergroup of $H$ such that $K \not \subset Z(H)$ then $K$ is open; see 3.4.) Say $H$ is an $S P_{-*}^{*}$ hypergroup if it has property $S_{n}$ for some $n$.

Definition 3.2. Let $M_{h}(H)=\{\mu \in M(H):|\mu| Z(H)=0\}$, an $L$-subspace of $M(H)$. Note $M(H)=M(Z(H)) \oplus M_{h}(H)$. Let $\pi$ be the normbounded projection: $M(H) \rightarrow M(Z(H))$. For $\mu \in M(H)$ we write $\mu=$ $\pi \mu+\mu_{h}$, so $\mu_{h} \in M_{h}(H)$.

We will show that if $H$ is an $S P_{-}^{*}$ hypergroup and $m_{H}(Z(H))=0$ then $M_{h}(H)$ is an ideal in $M(H)$ and its annihilator in $\Delta_{H}$ is $\Delta_{H} \backslash \hat{H}$. Thus $\Delta_{H} \mid \hat{H}$ is isomorphic to $\Delta_{Z(H)}$. The case $m_{H}(Z(H))>0$ will also be discussed.

Proposition 3.3. Suppose $H$ is an SP-* hypergroup with property $S_{n}$ for some positive integer $n$ and $\mu \in M_{h}(H)$, then $\mu^{n} \in L^{1}(H)$, (note $\mu^{n}=\mu * \mu \cdots * \mu(n$ times $\left.)\right)$. 
Proof. First suppose $\mu \in M_{h}(H)$ has compact support $K$ with $Z(H) \cap K=\varnothing$. Then for $\phi \in \hat{H},|\hat{\mu}(\phi)|=\left|\int_{K} \bar{\phi} d \mu\right| \leqq\|\mu\| \sup _{K}|\phi|$. We claim $\mu^{n} \in L^{2}(H) \subset L^{1}(H)$; indeed $\sum_{\phi \in \hat{H}} c(\phi)\left|\left(\mu^{n}\right)^{\wedge}(\phi)\right|^{2}=\sum_{\phi} c(\phi)|\hat{\mu}(\phi)|^{2 n} \leqq$ $\|\mu\|^{2 n} \sum_{\phi} c(\phi)\left(\sup _{K}|\phi|\right)^{2 n}<\infty$. The set of such $\mu$ is norm-dense in $M_{h}(H)$ and the map $\mu \mapsto \mu^{n}$ is norm-continuous taking a dense subset of $M_{h}(H)$ into $L^{1}(H)$, a closed subspace of $M(H)$.

For $M_{h}(H)$ to be a nontrivial ideal it is necessary that $L^{1}(H) \subset$ $M_{h}(H)$. We present a lemma which gives several equivalent characterizations of this.

Lemma 3.4. Let $K$ be a subhypergroup of a compact $P^{*}$-hypergroup $H$. The following statements are equivalent:

(Recall $\left.K^{\perp}=\{\phi \in \hat{H}: \phi \mid K=1\}\right)$

(1) $K$ is open;

(2) $m_{H}(K)>0$;

(3) each hypercoset of $K^{\perp}$ is finite;

(4) some hypercoset of $K^{\perp}$ is finite;

(5) $m_{K}$ is a nonzero multiple of $m_{H} \mid K$.

Proof. We first observe that each of (3) and (4) is equivalent to $K^{\perp}$ being finite. It $K^{\perp}$ is finite then each hypercoset $\phi \cdot K^{\perp},(\phi \in \hat{H})$, is finite, since $\phi \psi$ has finite support in $\hat{H},(\psi \in \hat{H})$. Further $K^{\perp}$ is contained in the support of $\bar{\phi} \cdot\left(\phi \cdot K^{\perp}\right)$ for each $\phi \in \hat{H}$, so if some hypercoset is finite then $K^{\perp}$ is finite (for more details see 3.16 [1]).

(1) implies (2): Note that the support of $m_{H}$ is $H$, (3.2 [1]).

(2) implies (3): The characteristic function $\chi_{K} \in L^{2}(H)$ and $\left(\chi_{K}\right)^{\wedge}(\phi)=\int_{K} \bar{\phi} d m_{H}=m_{H}(K)>0$ for $\phi \in K^{\perp}$. But $\sum_{\phi \in \hat{H}} c\left(\chi_{K}\right)\left|(\phi)^{\wedge}(\phi)\right|^{2}<$ $\infty$, thus $K^{\perp}$ is finite, (since $c(\phi) \geqq 1$ ).

(3) implies (1) and (5): Recall $\left(m_{K}\right)^{\wedge}$ is 1 on $K^{\perp}$ and 0 off $K^{\perp}(3.14[1])$. Since $K^{\perp}$ is finite we have $m_{K}=f \cdot m_{H}$ where $f \in C(H)$; in fact $f \in \operatorname{sp} \hat{H}$. Since the support of $m_{H}$ is $H$ we see that $f \geqq 0$ and $f=0$ off $K$. We will show that $f$ is constant on $K$, which implies that $K$ is open and $m_{K}$ is a nonzero multiple of $m_{H} \mid K$. Since $f \cdot m_{H}$ is the invariant measure on $K$, the identity $\left(f \cdot m_{H}\right) * \mu=f \cdot m_{H}$ holds for each $\mu \in M_{p}(K)$, (1.12 [1]). By Proposition 3.4 [1] this implies that

$$
f(x)=\int_{K} R(x) f\left(y^{\prime}\right) d \mu(y), \quad(x \in K) .
$$

Thus $f(x)=R(x) f\left(y^{\prime}\right)$ for each $x, y \in K$. Let $a=\sup _{K} f$ and let $K_{1}=$ $\{x \in K: f(x)=a\}$. For $x \in K_{1}, y \in K, a=f(x)=R(x) f\left(y^{\prime}\right)=\int_{K} f d \lambda\left(x, y^{\prime}\right)$, 
but this implies that $f$ is constant with value $a$ on the support of $\lambda\left(x, y^{\prime}\right)$. Thus $K_{1}$ is a nonempty (closed) ideal in $K$, but $K$ is normal so $K_{1}=K$ and $f$ is constant on $K$.

(5) implies (2): Clear.

Note if $H$ is an $S P_{-}^{*}$ hypergroup and $x \in H \backslash Z(H)$ then

$$
\{\phi \in \hat{H}:|\phi(x)|=1\}
$$

is finite, so if $K$ is a subhypergroup of $H$ with $K \not \subset Z(H)$ then $K^{\perp}$ is finite implying $K$ is open (by 3.4).

The following will be needed for the case where $Z(H)$ is open in $H$.

LEMma 3.5. Suppose $K$ is an open subhypergroup of a compact $P^{*}$-hypergroup $H, \psi \in \hat{K}$ and $\mu \in M(H)$ with $|\mu| K=0$, then

$$
\sum\{c(\phi) \hat{\mu}(\phi): \phi \in \hat{H}, \phi \mid K=\psi\}=0,
$$

(note this is a sum over a (finite) hypercoset of $K^{\perp}$ ).

Proof. We will show that $\sum_{\phi \mid K=\psi} c(\phi) \phi$ is equal to a multiple of $\psi$ on $K$ and is zero off $K$. By Lemma 3.4 there exists $d \geqq 1$ such that $m_{K}=d m_{H} \mid K$. Let $f \in C(H)$ be defined by $f=\psi$ on $K$ and $f=0$ off $K$. Then $\hat{f}(\phi)=\int_{K} \bar{\phi} \psi d m_{H}=(1 / d) \int_{K} \bar{\phi} \psi d m_{K}$, so $\hat{f}(\phi)=(d c(\psi))^{-1}$ for $\phi \mid K=\psi$ and $\hat{f}(\phi)=0$ otherwise, $\left(\right.$ note $c(\psi)=\left(\int_{K}|\psi|^{2} d m_{K}\right)^{-1}$, see 3.17 [1]).

Thus $f \in \operatorname{sp} \hat{H}$ and is given by the series $(d c(\psi))^{-1} \sum_{\phi \mid K=\psi} c(\phi) \phi$. Now

$$
\begin{aligned}
0 & =\int_{H} \bar{f} d \mu=(d c(\psi))^{-1} \sum_{\phi \mid K=\psi} c(\phi) \int_{H} \bar{\phi} d \mu \\
& =(d c(\psi))^{-1} \sum_{\phi \mid K=\psi} c(\phi) \hat{\mu}(\phi) .
\end{aligned}
$$

For the following $H$ will be an $S P_{-} *$ hypergroup, and for notational convenience we will write $G$ for $Z(H)$.

Proposition 3.6. If $m_{H} G=0$ then the projection $\pi: M(H) \rightarrow M(G)$

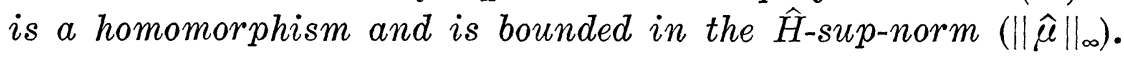

Proof. For $\mu \in M(H)$ we set $\mu=\pi \mu+\mu_{h}$. By 3.3 there exists an integer $n$ so that $\mu_{h}^{n} \in L^{1}(H)$. Thus $\hat{\mu}_{h} \rightarrow 0$ at $\infty$ on $\hat{H}$. Let $\gamma \in \hat{G}$, then $E_{r}=\{\phi \in \hat{H}: \phi \mid G=\gamma\}$ is a hypercoset of $G^{\perp}$ and is infinite (see 3.17 [1]). Let $\psi \in \kappa \hat{H} \backslash \hat{H}\left(\kappa \hat{H}\right.$ is the closure of $\hat{H}$ in $\Delta_{H}$ ) be the limit of an infinite convergent net $\left\{\phi_{\alpha}\right\} \subset E_{r}$. Then $\tilde{\mu}(\psi)=\lim _{\alpha} \tilde{\mu}\left(\phi_{\alpha}\right)=$ 
$\lim _{\alpha}\left((\pi \mu)^{\wedge}(\gamma)+\left(\mu_{h}\right)^{\wedge}\left(\phi_{\alpha}\right)\right)=(\pi \mu)^{\wedge}(\gamma)$. Note also $|\tilde{\mu}(\psi)| \leqq\|\hat{\mu}\|_{\infty}$. Thus $\left\|(\pi \mu)^{\wedge}\right\|_{\infty} \leqq\|\hat{\mu}\|_{\infty}$ and the functional $\mu \mapsto(\pi \mu)^{\wedge}(\gamma)$ is multiplicative for each $\gamma \in \hat{G}$. Hence $\pi$ is a homomorphism.

The following is now evident, (note for $\mu_{h} \in M_{h}(H)$ that $\tilde{\mu}_{h}=0$ off $\hat{H})$.

Theorem 3.7. If $m_{H} G=0$ then each element of $\Delta_{H} \backslash \hat{H}$ is of the form $\mu \mapsto(\pi \mu)^{\sim}(\psi)$ for some $\psi \in \Delta_{G}$. This correspondence is an isomorphism (of compact semitopological semigroups) of $\Delta_{H} \backslash \hat{H}$ with $\Delta_{G}$. The hypergroup $\kappa \hat{H}$ is isomorphic to $\hat{H} \cup \kappa \hat{G}$ (where $\kappa \hat{G}$ is the closure of $\widehat{G}$ in $\Delta_{G}$ ), and $\hat{H}$ is attached to $\kappa \hat{G}$ so that an unbounded net $\left\{\phi_{\alpha}\right\} \subset \hat{H}$ clusters at a point $\psi \in \kappa \widehat{G}$ if $\left\{\dot{\phi}_{\alpha} \mid G\right\} \subset \widehat{G}$ clusters at $\psi$.

In this particular situation, co $\Delta_{H}$ is already a semigroup. Let $S$ be the spectrum of the norm-closed span of $\Delta_{G}$ in $M(G)^{*}$, then $S$ is a compact semitopological semigroup (Taylor [7], or see [2, Ch. 1]). Let $\sigma_{1}$ be the canonical homomorphism: $M(G) \rightarrow M(S)$. Let $Y$ be the spectrum of the norm-closed span of co $\left(\Delta_{H}\right)$ in $M(H)^{*}$. Then $Y$ is the disjoint union of $H$ and $S$. The homomorphism $\sigma: M(H) \rightarrow M(Y)$ is given by $\sigma \mu=\sigma_{1}(\pi \mu)+\mu_{h}$; recall $\pi \mu \in M(G)$ so $\sigma_{1}(\pi \mu) \in M(S)$ and $\mu_{h} \in M(H)$. Since $\sigma$ has $w^{*}$-dense range we see that $H$ is an ideal in $Y$.

THEOREM 3.8. Suppose $m_{H} G=0$ and $\mu$ is an idempotent in $M(H)$, then $\pi \mu$ is an idempotent in $M(G)$ and $\hat{\mu}_{h}$ has finite support in $\hat{H}$. Thus $\{\phi \in \hat{H}: \hat{\mu}(\phi)=1\}$ is in the hypercoset ring of $\hat{H}$.

Proof. Since $\pi$ is a homomorphism, $\pi \mu$ is idempotent in $M(G)$. Thus $\left(\mu_{h}\right)^{\wedge}=\hat{\mu}-(\pi \mu)^{\wedge}$ is integer-valued, but tends to zero at $\infty$ on $\hat{H}$, so is zero for all but finitely many points in $\hat{H}$. By Cohen's theorem [2, Ch. 5], $S=\left\{\gamma \in \widehat{G}:(\pi \mu)^{\wedge}(\gamma)=1\right\}$ is in the coset ring of $\widehat{G}$. The set $\left\{\phi \in \hat{H}:(\pi \mu)^{\wedge}(\phi)=1\right\}=\{\phi \in \hat{H}: \phi \mid G \in S\}$, which is in the hypercoset ring of $\hat{H}$ (see $3.18[1])$.

If $G$ is open in $H$ then each hypercoset of $G^{\perp}$ is finite. In this case $M_{h}(H)$ is not an ideal (unless $H=G$ ), but $\mu \in M_{h}(H)$ does imply $\tilde{\mu}=0$ off $\hat{H}$. Each element of $\Delta_{I I} \mid \hat{H}$ is of the form $\mu \mapsto(\pi \mu)^{\sim}(\psi),(\mu \in M(H))$ for some $\psi \in \Delta_{G} \widehat{G}$. (Note if $\pi \mu \in L^{1}(G) \subset L^{1}(H)$ then $(\pi \mu)^{\sim}$ is zero off $\hat{G} \subset \Delta_{G}$ and is zero off $\hat{H} \subset \Delta_{H}$.) Thus $\Delta_{H} \backslash \hat{H}$ is isomorphic to $\Delta_{G} \mid \widehat{G}$. It can be shown that $\Delta_{H}$ is isomorphic to $\left(\Delta_{G} \mid \hat{G}\right) \cup \hat{H}$ with $\hat{H}$ attached to $\kappa \hat{G} \backslash \hat{G}$ in the obvious way.

THEOREM 3.7. If $G$ is open in $H$ and $\mu$ is an idempotent in $M(H)$ then $\{\phi \in \hat{H}: \hat{\mu}(\phi)=1\}$ is in the hypercoset ring of $\hat{H}$. 
Proof. Set $\mu=\pi \mu+\mu_{h}$. We will show $\hat{\mu}_{h}$ is finitely supported on $\hat{H}$, thus $\pi \mu$ differs from an idempotent in $M(G)$ by a trig polynomial on $G$ (an element of $\operatorname{sp} \hat{G} \subset C(G)$ ). Since $\hat{\mu}_{h} \rightarrow 0$ at $\infty$ on $\hat{H}$, the set $F=\left\{\phi \in \hat{H}:\left|\left(\mu_{h}\right)^{\wedge}(\phi)\right|>1 / 3\right\}$ is finite. Let $F_{1}=\bigcup_{\phi \in F} \phi \cdot G^{\perp}$, a finite union of hypercosets of $G^{\perp}$, then $F_{1}$ is finite since $G^{\perp}$ is finite (see 3.4). We claim $\left(\mu_{h}\right)^{\wedge}=0$ off $F_{1}$. Indeed, let $\phi \in \hat{H} \backslash F_{1}$ and suppose $\phi_{1} \in \hat{H}$ with $\phi\left|G=\phi_{1}\right| G$, then $\phi_{1} \notin F_{1}$ and $(\pi \mu)^{\wedge}(\phi)=(\pi \mu)^{\wedge}\left(\phi_{1}\right)$. Thus $\left|\hat{\mu}\left(\dot{\phi}_{1}\right)-\hat{\mu}(\phi)\right|=\left|\left(\mu_{h}\right)^{\wedge}\left(\phi_{1}\right)-\left(\mu_{h}\right)^{\wedge}(\phi)\right| \leqq 2 / 3$. But $\hat{\mu}$ is integer valued so $\hat{\mu}\left(\phi_{1}\right)=\hat{\mu}(\phi)$ and $\left(\mu_{h}\right)^{\wedge}\left(\phi_{1}\right)=\left(\mu_{h}\right)^{\wedge}(\phi)$. Thus $\widehat{\mu}_{h}$ is constant on $\phi \cdot G^{-}$and by Lemma 3.5 we have $\left(\mu_{h}\right)^{\wedge}=0$ on $\phi \cdot G^{-}$.

\section{REFERENCES}

1. C. DunkI, The measure algebra of a locally compact hypergroup, Trans. Amer. Math.

2. C. Dunkl and D. Ramirez, Topics in Harmonic Analysis, Appleton-Century-Crofts, New York, 1971.

3. I. Glicksberg, Convolution semigroups of measures, Pacific J. Math., 9 (1959), 51-67. 4. J. Pym, Weakly separately continuous measures algebras, Math. Annalen, 175 (1968), 207-219.

5. — Dual structures for measure algebras, Proc. London Math. Soc., (3), 19 (1969), 625-660.

6. D. Ragozin, Central measures on compact simple Lie groups, J. Functional Anal., 10 (1972), 212-229.

7. J. L. Taylor, The structure of convolution measure algebras, Trans. Amer. Math. Soc., 119 (1965), 150-166.

Received May 10, 1972. This research was partly supported by NSF Grant GP-31483X.

UNIVERSITY OF VIRGINIA 



\section{PACIFIC JOURNAL OF MATHEMATICS}

\section{EDITORS}

D. Gilbarg AND J. MILGRAM

Stanford University

Stanford, California 94305

\section{R. A. Beaumont}

University of Washington

Seattle, Washington 98105

\section{J. DUGUNDJI*}

Department of Mathematics University of Southern California Los Angeles, California 90007

RICHARD ARENS

University of California Los Angeles, California 90024

\section{ASSOCIATE EDITORS}
E. F. BECKENBACH
B. H. NEUMANN
F. WOLF
K. YoshidA

\section{SUPPORTING INSTITUTIONS}

\author{
UNIVERSITY OF BRITISH COLUMBIA \\ CALIFORNIA INSTITUTE OF TECHNOLOGY \\ UNIVERSITY OF CALIFORNIA \\ MONTANA STATE UNIVERSITY \\ UNIVERSITY OF NEVADA \\ NEW MEXICO STATE UNIVERSITY \\ OREGON STATE UNIVERSITY \\ UNIVERSITY OF OREGON \\ OSAKA UNIVERSITY
}

\author{
UNIVERSITY OF SOUTHERN CALIFORNIA \\ STANFORD UNIVERSITY \\ UNIVERSITY OF TOKYO \\ UNIVERSITY OF UTAH \\ WASHINGTON STATE UNIVERSITY \\ UNIVERSITY OF WASHINGTON \\ AMERICAN MATHEMATICAL SOCIETY \\ NAVAL WEAPONS CENTER
}

The Supporting Institutions listed above contribute to the cost of publication of this Journal, but they are not owners or publishers and have no responsibility for its content or policies.

Mathematical papers intended for publication in the Pacific Journal of Mathematics should be in typed form or offset-reproduced, (not dittoed), double spaced with large margins. Underline Greek letters in red, German in green, and script in blue. The first paragraph or two must be capable of being used separately as a synopsis of the entire paper. Items of the bibliography should not be cited there unless absolutely necessary, in which case they must be identified by author and Journal, rather than by item number. Manuscripts, in duplicate if possible, may be sent to any one of the four editors. Please classify according to the scheme of Math. Rev. Index to Vol. 39. All other communications to the editors should be addressed to the managing editor, Richard Arens, University of California, Los Angeles, California, 90024.

50 reprints are provided free for each article; additional copies may be obtained at cost in multiples of 50 .

The Pacific Journal of Mathematics is issued monthly as of January 1966. Regular subscription rate: $\$ 48.00$ a year (6 Vols., 12 issues). Special rate: $\$ 24.00$ a year to individual members of supporting institutions.

Subscriptions, orders for back numbers, and changes of address should be sent to Pacific Journal of Mathematics, 103 Highland Boulevard, Berkeley, California, 94708.

PUBLISHED BY PACIFIC JOURNAL OF MATHEMATICS, A NON-PROFIT CORPORATION

Printed at Kokusai Bunken Insatsusha (International Academic Printing Co., Ltd.), 270, 3-chome Totsuka-cho, Shinjuku-ku, Tokyo 160, Japan.

* C. DePrima will replace J. Dugundji until August 1974.

Copyright (C) 1973 by

Pacific Journal of Mathematics

All Rights Reserved 


\section{Pacific Journal of Mathematics}

\section{Vol. 47, No. $2 \quad$ February, 1973}

David Parham Bellamy, Composants of Hausdorff indecomposable continua; a mapping approach ........................ 303

Colin Bennett, A Hausdorff-Young theorem for rearrangement-invariant spaces ...........................................

Roger Daniel Bleier and Paul F. Conrad, The lattice of closed ideals and $a^{*}$-extensions of an abelian l-group ...

Ronald Elroy Bruck, Jr., Nonexpansive projections on subsets of Banach

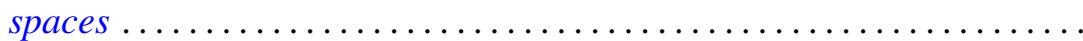

Robert C. Busby, Centralizers of twisted group algebras ............. 357

M. J. Canfell, Dimension theory in zero-set spaces ................ 393

John Dauns, One sided prime ideals ........................ 401

Charles F. Dunkl, Structure hypergroups for measure algebras . . . . . . . . . 413

Ronald Francis Gariepy, Geometric properties of Sobolev mappings ...... 427

Ralph Allen Gellar and Lavon Barry Page, A new look at some familiar spaces of intertwining operators ...........................

Dennis Michael Girard, The behavior of the norm of an automorphism of the

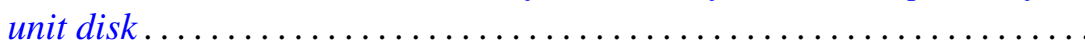

George Rudolph Gordh, Jr., Terminal subcontinua of hereditarily

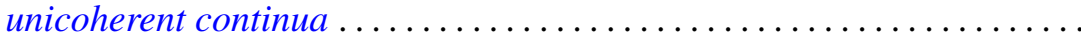

Joe Alston Guthrie, Mapping spaces and cs-networks. .

Neil Hindman, The product of $F$-spaces with $P$-spaces . 473

M. A. Labbé and John Wolfe, Isomorphic classes of the spaces $C_{\sigma}(S)$

Ernest A. Michael, On k-spaces, $k_{R}$-spaces and $k(X) \ldots$

Donald Steven Passman, Primitive group rings .

C. P. L. Rhodes, A note on primary decompositions of a pseudovaluation ...

Muril Lynn Robertson, A class of generalized functional differential equations

Ruth Silverman, Decomposition of plane convex sets. $I$.

Ernest Lester Stitzinger, On saturated formations of solvable Lie algebras................................

B. Andreas Troesch, Sloshing frequencies in a half-space by Kelvin inversion ...

L. E. Ward, Fixed point sets .

Michael John Westwater, Hilbert transforms, and a problem in scattering

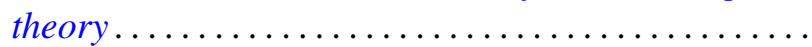

Misha Zafran, On the spectra of multipliers... 